\title{
Complex and Hypercomplex Discrete Fourier Transforms Based on Matrix Exponential Form of Euler's Formula
}

\author{
Stephen J. Sangwine* Todd A. Ell ${ }^{\dagger}$
}

July 5,2011

\begin{abstract}
We show that the discrete complex, and numerous hypercomplex, Fourier transforms defined and used so far by a number of researchers can be unified into a single framework based on a matrix exponential version of Euler's formula $e^{j \theta}=\cos \theta+j \sin \theta$, and a matrix root of -1 isomorphic to the imaginary root $j$. The transforms thus defined can be computed using standard matrix multiplications and additions with no hypercomplex code, the complex or hypercomplex algebra being represented by the form of the matrix root of -1 , so that the matrix multiplications are equivalent to multiplications in the appropriate algebra. We present examples from the complex, quaternion and biquaternion algebras, and from Clifford algebras $C \ell_{1,1}$ and $C \ell_{2,0}$. The significance of this result is both in the theoretical unification, and also in the scope it affords for insight into the structure of the various transforms, since the formulation is such a simple generalization of the classic complex case. It also shows that hypercomplex discrete Fourier transforms may be computed using standard matrix arithmetic packages without the need for a hypercomplex library, which is of importance in providing a reference implementation for verifying implementations based on hypercomplex code.
\end{abstract}

\section{Introduction}

The discrete Fourier transform is widely known and used in signal and image processing, and in many other fields where data is analyzed for frequency content [1]. The discrete Fourier transform in one dimension is classically formulated as:

$$
\begin{aligned}
& F[u]=S \sum_{m=0}^{M-1} f[m] \exp \left(-j 2 \pi \frac{m u}{M}\right) \\
& f[m]=T \sum_{u=0}^{M-1} F[u] \exp \left(j 2 \pi \frac{m u}{M}\right)
\end{aligned}
$$

where $j$ is the imaginary root of $-1, f[m]$ is real or complex valued with $M$ samples, $F[u]$ is complex valued, also with $M$ samples, and the two scale factors $S$ and $T$ must multiply to $1 / M$. If the transforms are to be unitary then $S$ must equal $T$ also. In this paper we discuss the formulation of the transform using a matrix exponential form of Euler's formula in which the imaginary square root of -1 is replaced by an isomorphic matrix root. This formulation works for the complex DFT, but more importantly, it works for hypercomplex DFTs (reviewed in $\S 2$ ). The matrix exponential formulation is equivalent to all the known hypercomplex generalizations of the DFT known to the authors, based on quaternion, biquaternion or Clifford algebras, through a suitable choice of matrix root of -1 , isomorphic to a root of -1 in the corresponding hypercomplex algebra. All associative hypercomplex algebras (and indeed the complex algebra) are known to be isomorphic

*Stephen J. Sangwine is with the School of Computer Science and Electronic Engineering, University of Essex, Wivenhoe Park, Colchester, CO4 3SQ, United Kingdom. Email: sjs@essex.ac.uk

$\dagger$ Todd A. Ell resides at 5620 Oak View Court, Savage, MN 55378-4695, USA. Email: T.Ell@IEEE.org 
to matrix algebras over the reals or the complex numbers. For example, Ward [2, § 2.8] discusses isomorphism between the quaternions and $4 \times 4$ real or $2 \times 2$ complex matrices so that quaternions can be replaced by matrices, the rules of matrix multiplication then being equivalent to the rules of quaternion multiplication by virtue of the pattern of the elements of the quaternion within the matrix. Also in the quaternion case, Ickes [3] wrote an important paper showing how multiplication of quaternions could be accomplished using a matrix-vector or vector-matrix product that could accommodate reversal of the product ordering by a partial transposition within the matrix. This paper, more than any other, led us to the observations presented here.

The fact that a hypercomplex DFT may be formulated using a matrix exponential may not be surprising. Nevertheless, to our knowledge, those who have worked on hypercomplex DFTs have not so far noted or exploited the observations made in this paper, which is surprising, given the ramifications discussed later.

\section{Hypercomplex transforms}

The first published descriptions of hypercomplex transforms that we are aware of date from the late 1980s, using quaternions. In all three known earliest formulations, the transforms were defined for two-dimensional signals (that is functions of two independent variables). The two earliest formulations [4, §6.4.2] and [5, Eqn. 20] are almost equivalent (they differ only in the placing of the exponentials and the signal and the signs inside the exponentials $)^{1}$ :

$$
F\left(\omega_{1}, \omega_{2}\right)=\int_{-\infty}^{\infty} \int_{-\infty}^{\infty} f\left(t_{1}, t_{2}\right) e^{i \omega_{1} t_{1}} e^{j \omega_{2} t_{2}} \mathrm{~d} t_{1} \mathrm{~d} t_{2}
$$

In a non-commutative algebra the ordering of exponentials within an integral is significant, and of course, two exponentials with different roots of -1 cannot be combined trivially. Therefore there are other possible transforms that can be defined by positioning the exponentials differently. The first transform in which the exponentials were placed either side of the signal function was that of Ell [6, 7]:

$$
F\left(\omega_{1}, \omega_{2}\right)=\int_{-\infty}^{\infty} \int_{-\infty}^{\infty} e^{i \omega_{1} t_{1}} f\left(t_{1}, t_{2}\right) e^{j \omega_{2} t_{2}} \mathrm{~d} t_{1} \mathrm{~d} t_{2}
$$

This style of transform was followed by Chernov, Bülow and Sommer [8, 9, 10] and others since. In 1998 the present authors described a single-sided hypercomplex transform for the first time [11 exactly as in (11) except that $f$ and $F$ were quaternion-valued and $j$ was replaced by a general quaternion root of -1 . Expressed in the same form as the transforms above, this would be:

$$
F\left(\omega_{1}, \omega_{2}\right)=\int_{-\infty}^{\infty} \int_{-\infty}^{\infty} e^{\boldsymbol{\mu}\left(\omega_{1} t_{1}+\omega_{2} t_{2}\right)} f\left(t_{1}, t_{2}\right) \mathrm{d} t_{1} \mathrm{~d} t_{2}
$$

where $\boldsymbol{\mu}$ is now an arbitrary root of -1 , not necessarily a basis element of the algebra. The realisation that an arbitrary root of -1 could be used meant that it was possible to define a hypercomplex transform applicable to one dimension:

$$
F(\omega)=\int_{-\infty}^{\infty} e^{\mu \omega t} f(t) \mathrm{d} t
$$

Pei et al have studied efficient implementation of quaternion FFTs and presented a transform based on commutative reduced biquaternions [12, 13. Ebling and Scheuermann defined a Clifford Fourier transform [14, $\S 5.2$ ], but their transform used the pseudoscalar (one of the basis elements of the algebra) as the square root of -1 .

Apart from the works by the present authors [11, 15, 16, the idea of using a root of -1 different to the basis elements of a hypercomplex algebra was not developed further until 2006, with the publication of

\footnotetext{
${ }^{1}$ In comparing the various formulations of hypercomplex transforms, we have changed the symbols used by the original authors in order to make the comparisons clearer. We have also made trivial changes such as the choice of basis elements used in the exponentials.
} 
a paper setting out the roots of -1 in biquaternions (a quaternion algebra with complex numbers as the components of the quaternions) [17. This work prepared the ground for a biquaternion Fourier transform [18 based on the present authors' one-sided quaternion transform [11. More recently, the idea of finding roots of -1 in other algebras has been advanced in Clifford algebras by Hitzer and Abłamowicz [19] with the express intent of using them in Clifford Fourier transforms, perhaps generalising the ideas of Ebling and Scheuermann [14. Finally, in this very brief summary of prior work we mention that the idea of applying hypercomplex algebras in signal processing has been studied by other authors apart from those referenced above. For an overview see [20].

In what follows we concentrate on DFTs in one dimension for simplicity, returning to the two dimensional case in $\S$,

\section{Matrix formulation of the discrete Fourier transform}

\subsection{Matrix form of Euler's formula}

The transform presented in this paper depends on a generalization of Euler's formula: $\exp i \theta=\cos \theta+i \sin \theta$, in which the imaginary root of -1 is replaced by a matrix root, that is, a matrix that squares to give a negated identity matrix. Even among $2 \times 2$ matrices there is an infinite number of such roots [21, p16]. In the matrix generalization, the exponential must, of course, be a matrix exponential [22, $\S 11.3]$. The following Lemma is not claimed to be original but we have not been able to locate any published source that we could cite here. Since the result is essential to Theorem 1 we set it out in full.

Lemma 1. Euler's formula may be generalized as follows:

$$
e^{\boldsymbol{J} \theta}=\boldsymbol{I} \cos \theta+\boldsymbol{J} \sin \theta
$$

where $\boldsymbol{I}$ is an identity matrix, and $\boldsymbol{J}^{2}=-\boldsymbol{I}$.

Proof. The result follows from the series expansions of the matrix exponential and the trigonometric functions. From the definition of the matrix exponential [22, §11.3]:

$$
e^{\boldsymbol{J} \theta}=\sum_{k=0}^{\infty} \frac{\boldsymbol{J}^{k} \theta^{k}}{k !}=\boldsymbol{J}^{0}+\boldsymbol{J} \theta+\frac{\boldsymbol{J}^{2} \theta^{2}}{2 !}+\frac{\boldsymbol{J}^{3} \theta^{3}}{3 !}+\cdots
$$

Noting that $\boldsymbol{J}^{0}=\boldsymbol{I}$ (see [23, Index Laws]), and separating the series into even and odd terms:

$$
\begin{aligned}
= & \boldsymbol{I}-\frac{\boldsymbol{I} \theta^{2}}{2 !}+\frac{\boldsymbol{I} \theta^{4}}{4 !}-\frac{\boldsymbol{I} \theta^{6}}{6 !}+\cdots \\
& +\boldsymbol{J} \theta-\frac{\boldsymbol{J} \theta^{3}}{3 !}+\frac{\boldsymbol{J} \theta^{5}}{5 !}-\frac{\boldsymbol{J} \theta^{7}}{7 !}+\cdots \\
= & \boldsymbol{I} \cos \theta+\boldsymbol{J} \sin \theta
\end{aligned}
$$

Note that matrix versions of the trigonometric functions are not needed to compute the matrix exponential, because $\theta$ is a scalar. In fact, if the exponential is evaluated numerically using a matrix exponential algorithm or function, the trigonometric functions are not even explicitly evaluated [22, § 11.3]. In practice, given that this is a special case of the matrix exponential, (because $\boldsymbol{J}^{2}=-\boldsymbol{I}$ ), it is likely to be numerically preferable to evaluate the trigonometric functions and to sum scaled versions of $\boldsymbol{I}$ and $\boldsymbol{J}$.

Notice that the matrix $e^{J \theta}$ has a structure with the cosine of $\theta$ on the diagonal and the (scaled) sine of $\theta$ where there are non-zero elements of $\boldsymbol{J}$. 


\subsection{Matrix form of DFT}

The classic discrete Fourier transform of (11) may be generalized to a matrix form in which the signals are vector-valued with $N$ components each and the root of -1 is replaced by an $N \times N$ matrix root $\boldsymbol{J}$ such that $\boldsymbol{J}^{2}=-\boldsymbol{I}$. In this form, subject to choosing the correct representation for the matrix root of -1 , we may represent a wide variety of complex and hypercomplex Fourier transforms.

Theorem 1. The following are a discrete Fourier transform pair ${ }^{2}$ :

$$
\begin{aligned}
& \boldsymbol{F}[:, u]=S \sum_{m=0}^{M-1} \exp \left(-\boldsymbol{J} 2 \pi \frac{m u}{M}\right) \boldsymbol{f}[:, m] \\
& \boldsymbol{f}[:, m]=T \sum_{u=0}^{M-1} \exp \left(\boldsymbol{J} 2 \pi \frac{m u}{M}\right) \boldsymbol{F}[:, u]
\end{aligned}
$$

where $\boldsymbol{J}$ is a $N \times N$ matrix root of $-1, \boldsymbol{f}$ and $\boldsymbol{F}$ are $N \times M$ matrices with one sample per column, and the two scale factors $S$ and $T$ multiply to give $1 / M$.

Proof. The proof is based on substitution of the forward transform (2) into the inverse (3) followed by algebraic reduction to a result equal to the original signal $f$. We start by substituting (2) into (3), replacing $m$ by $\mathcal{M}$ to keep the two indices distinct, and at the same time replacing the two scale factors by their product $1 / M$ :

$$
\boldsymbol{f}[:, m]=\frac{1}{M} \sum_{u=0}^{M-1}\left[e^{J 2 \pi \frac{m u}{M}} \sum_{\mathcal{M}=0}^{M-1} e^{-J 2 \pi \frac{\mathcal{M} u}{M}} \boldsymbol{f}[:, \mathcal{M}]\right]
$$

The exponential of the outer summation can be moved inside the inner, because it is constant with respect to the summation index $\mathcal{M}$ :

$$
\boldsymbol{f}[:, m]=\frac{1}{M} \sum_{u=0}^{M-1} \sum_{\mathcal{M}=0}^{M-1} e^{J 2 \pi \frac{m u}{M}} e^{-\boldsymbol{J} 2 \pi \frac{\mathcal{M} u}{M}} \boldsymbol{f}[:, \mathcal{M}]
$$

The two exponentials have the same root of -1 , namely $\boldsymbol{J}$, and therefore they can be combined:

$$
\boldsymbol{f}[:, m]=\frac{1}{M} \sum_{u=0}^{M-1} \sum_{\mathcal{M}=0}^{M-1} e^{J 2 \pi \frac{(m-\mathcal{M}) u}{M}} \boldsymbol{f}[:, \mathcal{M}]
$$

We now isolate out from the inner summation the case where $m=\mathcal{M}$. In this case the exponential reduces to an identity matrix, and we have:

$$
\begin{aligned}
\boldsymbol{f}[:, m] & =\frac{1}{M} \sum_{u=0}^{M-1} \boldsymbol{f}[:, m] \\
& +\frac{1}{M} \sum_{u=0}^{M-1}\left[\left.\sum_{\mathcal{M}=0}^{M-1}\right|_{\mathcal{M} \neq m} e^{\left.J \frac{2 \pi \frac{(m-\mathcal{M}) u}{M}}{f}[:, \mathcal{M}]\right]}\right.
\end{aligned}
$$

The first line on the right sums to $\boldsymbol{f}[:, m]$, which is the original signal, as required. To complete the proof, we have to show that the second line on the right reduces to zero. Taking the second line alone, and changing the order of summation, we obtain:

$$
\left.\sum_{\mathcal{M}=0}^{M-1}\right|_{\mathcal{M} \neq m}\left[\sum_{u=0}^{M-1} e^{J 2 \pi \frac{(m-\mathcal{M}) u}{M}}\right] \boldsymbol{f}[:, \mathcal{M}]
$$

\footnotetext{
${ }^{2}$ The colon notation used here will be familiar to users of MATLABß (an explanation may be found in [22, $\left.\S 1.1 .8\right]$ ). Briefly, $\boldsymbol{f}[:, m]$ means the $m^{\text {th }}$ column of the matrix $\boldsymbol{f}$.
} 
Using Lemma 1 we now write the matrix exponential as the sum of a cosine and sine term.

$$
\left.\sum_{\mathcal{M}=0}^{M-1}\right|_{m \neq \mathcal{M}}\left[\begin{array}{c}
\boldsymbol{I} \sum_{u=0}^{M-1} \cos \left(2 \pi \frac{(m-\mathcal{M}) u}{M}\right) \\
+\boldsymbol{J} \sum_{u=0}^{M-1} \sin \left(2 \pi \frac{(m-\mathcal{M}) u}{M}\right)
\end{array}\right] \boldsymbol{f}[:, \mathcal{M}]
$$

Since both of the inner summations are sinusoids summed over an integral number of cyles, they vanish, and this completes the proof.

Notice that the requirement for $\boldsymbol{J}^{2}=-\boldsymbol{I}$ is the only constraint on $\boldsymbol{J}$. It is not necessary to constrain elements of $\boldsymbol{J}$ to be real. Note that $\boldsymbol{J}^{2}=-\boldsymbol{I}$ implies that $\boldsymbol{J}^{-1}=-\boldsymbol{J}$, hence the inverse transform is obtained by negating or inverting the matrix root of -1 (the two operations are equivalent).

The matrix dimensions must be consistent according to the ordering inside the summation. As written above, for a complex transform represented in matrix form, $\boldsymbol{f}$ and $\boldsymbol{F}$ must have two rows and $M$ columns. If the exponential were to be placed on the right, $\boldsymbol{f}$ and $\boldsymbol{F}$ would have to be transposed, with two columns and $M$ rows.

It is important to realize that (2) is totally different to the classical matrix formulation of the discrete Fourier transform, as given for example by Golub and Van Loan [22, §4.6.4]. The classic DFT given in (11) can be formulated as a matrix equation in which a large $M \times M$ Vandermonde matrix containing $n^{\text {th }}$ roots of unity multiplies the signal $f$ expressed as a vector of real or complex values. Instead, in (2) each matrix exponential multiplies one column of $\boldsymbol{f}$, corresponding to one sample of the signal represented by $\boldsymbol{f}$ and the dimensions of the matrix exponential are set by the dimensionality of the algebra ( 2 for complex, 4 for quaternions etc.). In (2) it is the multiplication of the exponential and the signal samples, dependent on the algebra involved, that is expressed in matrix form, not the structure of the transform itself.

Readers who are already familiar with hypercomplex Fourier transforms should note that the ordering of the exponential within the summation (2) is not related to the ordering within the hypercomplex formulation of the transform (which is significant because of non-commutative multiplication). The hypercomplex ordering can be accommodated within the framework presented here by changing the representation of the matrix root of -1 , in a non-trivial way, shown for the quaternion case by Ickes [3, Equation 10] and called transmutation. We have studied the generalisation of Ickes' transmutation to the case of Clifford algebras, and it appears that there is a more general operation. In the cases we have studied this can be described as negation of the off-diagonal elements of the lower-right sub-matrix, excluding the first row and column ${ }^{3}$. We believe a more general result is known in Clifford algebra but we have not been able to locate a clear statement that we could cite. We therefore leave this for later work, as a full generalisation to Clifford algebras of arbitrary dimension requires further work, and is more appropriate to a more mathematical paper.

\section{Examples in specific algebras}

In this section we present the information necessary for (2) and (3) to be verified numerically. In each of the cases below, we present an example root of -1 and a matrix representation ${ }^{4}$. We include in the Appendix a short MATLAB@ function for computing the transform in (2). The same code will compute the inverse by negating $\boldsymbol{J}$. This may be used to verify the results in the next section and to compare the results obtained with the classic complex FFT. In order to verify the quaternion or biquaternion results, the reader will need to install the QTFM toolbox [24, or use some other specialised software for computing with quaternions.

\footnotetext{
${ }^{3}$ This gives the same result as transmutation in the quaternion case.

${ }^{4}$ The matrix representations of roots of -1 are not unique - a transpose of the matrix, for example, is equally valid. The operations that leave the square of the matrix invariant probably correspond to fundamental operations in the hypercomplex algebra, for example negation, conjugation, reversion.
} 


\subsection{Complex algebra}

The $2 \times 2$ real matrix $\left(\begin{array}{rr}0 & -1 \\ 1 & 0\end{array}\right)$ can be easily verified by eye to be a square root of the negated identity matrix $\left(\begin{array}{rr}-1 & 0 \\ 0 & -1\end{array}\right)$, and it is easy to verify numerically that Euler's formula gives the same numerical results in the classic complex case and in the matrix case for an arbitrary $\theta$. This root of -1 is based on the well-known isomorphism between a complex number $a+j b$ and the matrix representation $\left(\begin{array}{cc}a & -b \\ b & a\end{array}\right)$ [2, Theorem 1.6 $]^{5}$. The structure of a matrix exponential $e^{\boldsymbol{J} \theta}$ using the above matrix for $\boldsymbol{J}$ is $\left(\begin{array}{cc}C & -S \\ S & C\end{array}\right)$ where $C=\cos \theta$ and $S=\sin \theta$.

\subsection{Quaternion algebra}

The quaternion roots of -1 were discovered by Hamilton [25, pp 203, 209], and consist of all unit pure quaternions, that is quaternions of the form $x \boldsymbol{i}+y \boldsymbol{j}+z \boldsymbol{k}$ subject to the constraint $x^{2}+y^{2}+z^{2}=1$. A simple example is the quaternion $\boldsymbol{\mu}=(\boldsymbol{i}+\boldsymbol{j}+\boldsymbol{k}) / \sqrt{3}$, which can be verified by hand to be a square root of -1 using the usual rules for multiplying the quaternion basis elements $\left(\boldsymbol{i}^{2}=\boldsymbol{j}^{2}=\boldsymbol{k}^{2}=\boldsymbol{i} \boldsymbol{j} \boldsymbol{k}=-1\right)$. Using the isomorphism with $4 \times 4$ matrices given by Ward [2, $\S 2.8$ ], between the quaternion $w+x \boldsymbol{i}+y \boldsymbol{j}+z \boldsymbol{k}$ and the matrix:

$$
\left(\begin{array}{rrrr}
w & -x & -y & -z \\
x & w & -z & y \\
y & z & w & -x \\
z & -y & x & w
\end{array}\right)
$$

we have the following matrix representation:

$$
\boldsymbol{\mu}=\frac{1}{\sqrt{3}}\left(\begin{array}{rrrr}
0 & -1 & -1 & -1 \\
1 & 0 & -1 & 1 \\
1 & 1 & 0 & -1 \\
1 & -1 & 1 & 0
\end{array}\right)
$$

Notice the structure that is apparent in this matrix: the $2 \times 2$ blocks on the leading diagonal at the top left and bottom right can be recognised as roots of -1 in the complex algebra as shown in $\S 4.1$

Proposition 1. Any matrix of the form:

$$
\left(\begin{array}{rrrr}
0 & -x & -y & -z \\
x & 0 & -z & y \\
y & z & 0 & -x \\
z & -y & x & 0
\end{array}\right)
$$

with $x^{2}+y^{2}+z^{2}=1$ is the square root of a negated $4 \times 4$ identity matrix. Thus the matrix representations of the quaternion roots of -1 are all roots of the negated $4 \times 4$ identity matrix.

Proof. The matrix is anti-symmetric, and the inner product of the $i^{\text {th }}$ row and $i^{\text {th }}$ column is $-x^{2}-y^{2}-z^{2}$, which is -1 because of the stated constraint. Therefore the diagonal elements of the square of the matrix are -1 . Note that the rows of the matrix have one or three negative values, whereas the columns have zero or two. The product of the $i^{\text {th }}$ row with the $j^{\text {th }}$ column, $i \neq j$, is the sum of two values of opposite sign and equal magnitude. Therefore all off-diagonal elements of the square of the matrix are zero.

The structure of a matrix exponential $e^{J \theta}$ using a matrix as in Proposition 1 for $\boldsymbol{J}$ is:

$$
\left(\begin{array}{rrrr}
C & -x S & -y S & -z S \\
x S & C & -z S & y S \\
y S & z S & C & -x S \\
z S & -y S & x S & C
\end{array}\right)
$$

\footnotetext{
${ }^{5}$ We have used the transpose of Ward's representation for consistency with the quaternion and biquaternion representations in the two following sections.
} 
where, as before, $C=\cos \theta$ and $S=\sin \theta$.

\subsection{Biquaternion algebra}

The biquaternion algebra [2, Chapter 3] (quaternions with complex elements) can be handled exactly as in the previous section, except that the $4 \times 4$ matrix representing the root of -1 must be complex (and the signal matrix must have four complex elements per column). The set of square roots of -1 in the biquaternion algebra is given in [17. A simple example is $\boldsymbol{i}+\boldsymbol{j}+\boldsymbol{k}+I(\boldsymbol{j}-\boldsymbol{k})$ (where $I$ denotes the classical complex root of -1 , that is the biquaternion has real part $\boldsymbol{i}+\boldsymbol{j}+\boldsymbol{k}$ and imaginary part $\boldsymbol{j}-\boldsymbol{k}$ ). Again, this can be verified by hand to be a root of -1 and its matrix representation is:

$$
\left(\begin{array}{cccc}
0 & -1 & -1-I & -1+I \\
1 & 0 & -1+I & 1+I \\
1+I & 1-I & 0 & -1 \\
1-I & -1-I & 1 & 0
\end{array}\right)
$$

Again, sub-blocks of the matrix have recognizable structure. The upper left and lower right diagonal $2 \times 2$ blocks are roots of -1 , while the lower left and upper right off-diagonal $2 \times 2$ blocks are nilpotent - that is their square vanishes.

\subsection{Clifford algebras}

Recent work by Hitzer and Abłamowicz [19] has explored the roots of -1 in Clifford algebras $C \ell_{p, q}$ up to those with $p+q=4$, which are 16-dimensional algebras ${ }^{6}$. The derivations of the roots of -1 for the 16 dimensional algebras are long and difficult. Therefore, for the moment, we confine the discussion here to lower-order algebras, noting that, since all Clifford algebras are isomorphic to a matrix algebra, we can be assured that if roots of -1 exist, they must have a matrix representation. Using the results obtained by Hitzer and Abłamowicz, and by finding from first principles the layout of a real matrix isomorphic to a Clifford multivector in a given algebra, it has been possible to verify that the transform formulation presented in this paper is applicable to at least the lower order Clifford algebras. The quaternions and biquaternions are isomorphic to the Clifford algebras $C \ell_{0,2}$ and $C \ell_{3,0}$ respectively so this is not surprising. Nevertheless, it is an important finding, because until now quaternion and Clifford Fourier transforms were defined in different ways, using different terminology, and it was difficult to make comparisons between the two. Now, with the matrix exponential formulation, it is possible to handle quaternion and Clifford transforms (and indeed transforms in different Clifford algebras) within the same algebraic and/or numerical framework.

We present examples here from two of the 4-dimensional Clifford algebras, namely $C \ell_{1,1}$ and $C \ell_{2,0}$. These results have been verified against the CLICAL package [26] to ensure that the multiplication rules have been followed correctly and that the roots of -1 found by Hitzer and Abłamowicz are correct.

Following the notation in [19, we write a multivector in $C \ell_{1,1}$ as $\alpha+b_{1} e_{1}+b_{2} e_{2}+\beta e_{12}$, where $e_{1}^{2}=$ $+1, e_{2}^{2}=-1, e_{12}^{2}=+1$ and $e_{1} e_{2}=e_{12}$. A possible real matrix representation is as follows:

$$
\left(\begin{array}{rrrr}
\alpha & b_{1} & -b_{2} & \beta \\
b_{1} & \alpha & -\beta & b_{2} \\
b_{2} & -\beta & \alpha & b_{1} \\
\beta & -b_{2} & b_{1} & \alpha
\end{array}\right)
$$

In this algebra, the constraints on the coefficients of a multivector for it to be a root of -1 are as follows: $\alpha=0$ and $b_{1}^{2}-b_{2}^{2}+\beta^{2}=-1[19 \text {, Table } 1]^{7}$. Choosing $b_{1}=\beta=1$ gives $b_{2}=\sqrt{3}$ and thus $e_{1}+\sqrt{3} e_{2}+e_{12}$

\footnotetext{
${ }^{6} p$ and $q$ are non-negative integers such that $p+q=n$ and $n \geq 1$. The dimension of the algebra (strictly the dimension of the space spanned by the basis elements of the algebra) is $2^{n}$.

${ }^{7}$ We have re-arranged the constraint compared to [19] Table 1] to make the comparison with the quaternion case easier: we see that the signs of the squares of the coefficients match the signs of the squared basis elements.
} 
which can be verified algebraically or in CLICAL to be a root of -1 . The corresponding matrix is then:

$$
\left(\begin{array}{cccc}
0 & 1 & -\sqrt{3} & 1 \\
1 & 0 & -1 & \sqrt{3} \\
\sqrt{3} & -1 & 0 & 1 \\
1 & -\sqrt{3} & 1 & 0
\end{array}\right)
$$

Following the same notation in algebra $C \ell_{2,0}$, in which $e_{1}^{2}=e_{2}^{2}=+1, e_{12}^{2}=-1$, a possible matrix representation is:

$$
\left(\begin{array}{rrrr}
\alpha & b_{1} & b_{2} & -\beta \\
b_{1} & \alpha & \beta & -b_{2} \\
b_{2} & -\beta & \alpha & b_{1} \\
\beta & -b_{2} & b_{1} & \alpha
\end{array}\right)
$$

The constraints on the coefficients are $\alpha=0$ and $b_{1}^{2}+b_{2}^{2}-\beta^{2}=-1$, and choosing $b_{1}=b_{2}=1$ gives $\beta=\sqrt{3}$ and thus $e_{1}+e_{2}+\sqrt{3} e_{12}$ is a root of -1 . The corresponding matrix is then:

$$
\left(\begin{array}{cccc}
0 & 1 & 1 & -\sqrt{3} \\
1 & 0 & \sqrt{3} & -1 \\
1 & -\sqrt{3} & 0 & 1 \\
\sqrt{3} & -1 & 1 & 0
\end{array}\right)
$$

Notice that in both of these algebras the matrix representation of a root of -1 is very similar to that given for the quaternion case in Proposition 1, with zeros on the leading diagonal, an odd number of negative values in each row and an even number in each column. It is therefore simple to see that minor modifications to Proposition 1 would cover these algebras and the matrices presented above.

\section{$5 \quad$ An example not based on a specific algebra}

We show here using an arbitrary $2 \times 2$ matrix root of -1 , that it is possible to define a Fourier transform without a specific algebra. Let an arbitrary real matrix be given as $J=\left(\begin{array}{ll}a & b \\ c & d\end{array}\right)$, then by brute force expansion of $J^{2}=-I$ we find the original four equations reduce to but two independent equations. Picking $(a, b)$ and solving for the remaining coefficients we find that any matrix of the form:

$$
\left(\begin{array}{cr}
a & b \\
-\left(1+a^{2}\right) / b & -a
\end{array}\right)
$$

with finite $a$ and $b$, and $b \neq 0$, is a root of -1 . Choosing instead $(a, c)$ we get the transpose form:

$$
\left(\begin{array}{cc}
a & -\left(1+a^{2}\right) / c \\
c & -a
\end{array}\right)
$$

where $c \neq 0$. Choosing the cross-diagonal terms $(b, c)$ yields:

$$
\left(\begin{array}{cc} 
\pm \kappa & b \\
c & \mp \kappa
\end{array}\right)
$$

where $\kappa=\sqrt{-1-b c}$ and $b c \leq-1$.

In all cases the resulting matrix has eigenvalues of $\lambda= \pm i$. (This is a direct consequence of the fact that this matrix squares to -1.) Each form, however, has different eigenvectors. The standard matrix representation for the complex operator $i$ is $\left(\begin{array}{rr}0 & -1 \\ 1 & 0\end{array}\right)$ with eigenvectors $v=[1, \pm i]$. In the matrix with $(a, b)$ 
parameters the eigenvectors are $v=[1,-b /(a \pm i)]$ whereas the cross-diagonal form with $(b, c)$ parameters has eigenvectors $v=[1,(\kappa \pm i) / c]$.

These forms suggest the interesting question: which algebra, if any, applies here ${ }^{8}$; and how can the Fourier coefficients (the 'spectrum') be interpreted? We are not able to answer the first question in this paper. The 'interpretation' of the spectrum is relatively simple. Consider a spectrum $\boldsymbol{F}$ containing only one non-zero column at index $u_{0}$ with value $\left(\begin{array}{l}x \\ y\end{array}\right)$ and invert this spectrum using (3). Ignoring the scale factor, the result will be the signal:

$$
\boldsymbol{f}[:, m]=\exp \left(\boldsymbol{J} 2 \pi \frac{m u_{0}}{M}\right)\left(\begin{array}{l}
x \\
y
\end{array}\right)
$$

The form of the matrix exponential depends on $\boldsymbol{J}$. In the classic complex case, as given in $\S 4.1$, the matrix exponential, as already seen, takes the form:

$$
\left(\begin{array}{rr}
\cos \theta & -\sin \theta \\
\sin \theta & \cos \theta
\end{array}\right)
$$

where $\theta=2 \pi \frac{m u_{0}}{M}$. This is a rotation matrix and it maps a real unit vector $\left(\begin{array}{l}1 \\ 0\end{array}\right)$ to a point on a circle in the complex plane. It embodies the standard phasor concept associated with sinusoidal functions. Using the same analysis, this time using the matrix in (44) above, one obtains for the matrix exponential the 'phasor' operator:

$$
\left(\begin{array}{rr}
\cos \theta+\kappa \sin \theta & b \sin \theta \\
c \sin \theta & \cos \theta-\kappa \sin \theta
\end{array}\right)
$$

Instead of mapping a real unit vector $\left(\begin{array}{l}1 \\ 0\end{array}\right)$ to a point on a circle, this matrix maps to an ellipse. Thus, we see that a transform based on a matrix such as that in (40) has basis functions that are projections of an elliptical, rather than a circular path in the complex plane, as in the classical complex Fourier transform. We refer the reader to a discussion on a similar point for the one-sided quaternion discrete Fourier transform in our own 2007 paper [16, § VI], in which we showed that the quaternion coefficients of the Fourier spectrum also represent elliptical paths through the space of the signal samples.

It is possible that the matrices discussed in this section could be transformed by similarity transformations into matrices representing elements of a Clifford algebra ${ }^{9}$. Note that in the quaternion case, any root of -1 lies on the unit sphere in 3-space, and can therefore be transformed into another root of -1 by a rotation. It is possible that the same applies in other algebras, the transformation needed being dependent on the geometry. Clearly there are interesting issues to be studied here, and further work to be done.

\section{Non-existence of transforms in algebras with odd dimension}

In this section we show that there are no real matrix roots of -1 with odd dimension. This is not unexpected, since the existence of such roots would suggest the existence of a hypercomplex algebra of odd dimension. The significance of this result is to show that there is no discrete Fourier transform as formulated in Theorem 1 for an algebra of dimension 3, which is of importance for the processing of signals representing physical 3 -space quantities, or the values of colour image pixels. We thus conclude that the choice of quaternion Fourier transforms or a Clifford Fourier transform of dimension 4 is inevitable in these cases. This is not an unexpected conclusion, nevertheless, in the experience of the authors, some researchers in signal and image processing hesitate to accept the idea of using four dimensions to handle three-dimensional samples or pixels. (This is despite the rather obvious parallel of needing two dimensions - complex numbers - to represent the Fourier coefficients of a real-valued signal or image.)

Theorem 2. There are no $N \times N$ matrices $\boldsymbol{J}$ with real elements such that $\boldsymbol{J}^{2}=-\boldsymbol{I}$ for odd values of $N$.

\footnotetext{
${ }^{8}$ It is possible that there is no corresponding 'algebra' in the usual sense. Note that there are only two Clifford algebras of dimension 2, one of which is the algebra of complex numbers. The other has no multivector roots of -1 [19] $\S 4$ ] and therefore the roots of -1 given above cannot be a root of -1 in any Clifford algebra.

${ }^{9}$ We are grateful to Dr Eckhard Hitzer for pointing this out, in September 2010.
} 
Proof. The determinant of a diagonal matrix is the product of its diagonal entries. Therefore $|-\boldsymbol{I}|=-1$ for odd $N$. Since the product of two determinants is the determinant of the product, $\left|\boldsymbol{J}^{2}\right|=-1$ requires $|\boldsymbol{J}|^{2}=-1$, which cannot be satisfied if $\boldsymbol{J}$ has real elements.

\section{Extension to two-sided DFTs}

There have been various definitions of two sided hypercomplex Fourier transforms and DFTs. We consider here only one case to demonstrate that the approach presented in this paper is applicable to two-sided as well as one-sided transforms: this is a matrix exponential Fourier transform based on Ell's original two-sided two-dimensional quaternion transform [6, Theorem 4.1], 7], 27]. A more general formulation is:

$$
\begin{aligned}
& \boldsymbol{F}[u, v]=S \sum_{m=0}^{M-1} \sum_{n=0}^{N-1} e^{-\boldsymbol{J} 2 \pi \frac{m u}{M}} \boldsymbol{f}[m, n] e^{-\boldsymbol{K} 2 \pi \frac{n v}{N}} \\
& \boldsymbol{f}[m, n]=T \sum_{u=0}^{M-1} \sum_{v=0}^{N-1} e^{+\boldsymbol{J} 2 \pi \frac{m u}{M}} \boldsymbol{F}[u, v] e^{+\boldsymbol{K} 2 \pi \frac{n v}{N}}
\end{aligned}
$$

in which each element of the two-dimensional arrays $\boldsymbol{F}$ and $\boldsymbol{f}$ is a square matrix representing a complex or hypercomplex number using a matrix isomorphism for the algebra in use, for example the representations already given in $\S 4.2$ in the case of the quaternion algebra; the two scale factors multiply to give $1 / M N$, and $\boldsymbol{J}$ and $\boldsymbol{K}$ are matrix representations of two arbitrary roots of -1 in the chosen algebra. (In Ell's original formulation, the roots of -1 were $\boldsymbol{j}$ and $\boldsymbol{k}$, that is two of the orthogonal quaternion basis elements. The following theorem shows that there is no requirement for the two roots to be orthogonal in order for the transform to invert.)

Theorem 3. The transforms in (5) and (6) are a two-dimensional discrete Fourier transform pair, provided that $\boldsymbol{J}^{2}=\boldsymbol{K}^{2}=-\boldsymbol{I}$.

Proof. The proof follows the same scheme as the proof of Theorem 1 but we adopt a more concise presentation to fit the available column space. We start by substituting (2) into (3), replacing $m$ and $n$ by $\mathcal{M}$ and $\mathcal{N}$ respectively to keep the indices distinct:

$$
\begin{aligned}
\boldsymbol{f}[m, n] & =\frac{1}{M N} \sum_{u=0}^{M-1} \sum_{v=0}^{N-1} e^{J 2 \pi \frac{m u}{M}} \\
& \times\left[\sum_{\mathcal{M}=0}^{M-1} \sum_{\mathcal{N}=0}^{N-1} e^{-\boldsymbol{J} 2 \pi \frac{\mathcal{M} u}{M}} \boldsymbol{f}[\mathcal{M}, \mathcal{N}] e^{-\boldsymbol{K} 2 \pi \frac{\mathcal{N} v}{N}}\right] \\
& \times e^{\boldsymbol{K} 2 \pi \frac{n v}{N}}
\end{aligned}
$$

The scale factors can be moved outside both summations, and replaced with their product $1 / M N$; and the exponentials of the outer summations can be moved inside the inner, because they are constant with respect to the summation indices $\mathcal{M}$ and $\mathcal{N}$. At the same time, adjacent exponentials with the same root of -1 can be merged. With these changes, and omitting the scale factor to save space, the right-hand side of the equation becomes:

$$
\sum_{u=0}^{M-1} \sum_{v=0}^{N-1} \sum_{\mathcal{M}=0}^{M-1} \sum_{\mathcal{N}=0}^{N-1} e^{J 2 \pi \frac{(m-\mathcal{M}) u}{M}} f[\mathcal{M}, \mathcal{N}] e^{K 2 \pi \frac{(n-\mathcal{N}) v}{N}}
$$

We now isolate out from the inner pair of summations the case where $\mathcal{M}=m$ and $\mathcal{N}=n$. In this case the exponentials reduce to identity matrices, and we have:

$$
\frac{1}{M N} \sum_{u=0}^{M-1} \sum_{v=0}^{N-1} \boldsymbol{f}[m, n]
$$


This sums to $\boldsymbol{f}[m, n]$, which is the original two-dimensional signal, as required. To complete the proof we have to show that the rest of the summation, excluding the case $\mathcal{M}=m$ and $\mathcal{N}=n$, reduces to zero. Dropping the scale factor, and changing the order of summation, we have the following inner double summation:

$$
\sum_{u=0}^{M-1} \sum_{v=0}^{N-1} e^{J 2 \pi \frac{(m-\mathcal{M}) u}{M}} \boldsymbol{f}[\mathcal{M}, \mathcal{N}] e^{K 2 \pi \frac{(n-\mathcal{N}) v}{N}}
$$

Noting that the first exponential and $\boldsymbol{f}$ are independent of the second summation index $v$, we can move them outside the second summation (we could do similarly with the exponential on the right and the first summation):

$$
\sum_{u=0}^{M-1} e^{J 2 \pi \frac{(m-\mathcal{M}) u}{M}} f[\mathcal{M}, \mathcal{N}] \sum_{v=0}^{N-1} e^{K 2 \pi \frac{(n-\mathcal{N}) v}{N}}
$$

and, as in Theorem 1 the summation on the right is over an integral number of cycles of cosine and sine, and therefore vanishes.

Notice that it was not necessary to assume that $\boldsymbol{J}$ and $\boldsymbol{K}$ were orthogonal: it is sufficient that each be a root of -1 . This has been verified numerically using the two-dimensional code given in the Appendix.

\section{Discussion}

We have shown that any discrete Fourier transform in an algebra that has a matrix representation, can be formulated in the way shown here. This includes the complex, quaternion, biquaternion, and Clifford algebras (although we have demonstrated only certain cases of Clifford algebras, we believe the result holds in general). This observation provides a theoretical unification of diverse hypercomplex DFTs.

Several immediate possibilities for further work, as well as ramifications, now suggest themselves. Firstly, the study of roots of -1 is accessible from the matrix representation as well as direct representation in whatever algebra is employed for the transform. All of the results obtained so far in hypercomplex algebras, and known to the authors [25, pp 203, 209], [17, 19, were achieved by working in the algebra in question, that is by algebraic manipulation of quaternion, biquaternion or Clifford multivector values. An alternative approach would be to work in the equivalent matrix algebra, but this seems difficult even for the lower order cases. Nevertheless, it merits further study because of the possibility of finding a systematic approach that would cover many algebras in one framework. Following the reasoning in $\S$, it is possible to define matrix roots of -1 that appear not to be isomorphic to any Clifford or quaternion algebra, and these merit further study.

Secondly, the matrix formulation presented here lends itself to analysis of the structure of the transform, including possible factorizations for fast algorithms, as well as parallel or vectorized implementations for single-instruction, multiple-data (SIMD) processors, and of course, factorizations into multiple complex FFTs as has been done for quaternion FFTs (see for example 15). In the case of matrix roots of -1 which do not correspond to Clifford or quaternion algebras, analysis of the structure of the transform may give insight into possible applications of transforms based on such roots.

Finally, at a practical level, hypercomplex transforms implemented directly in hypercomplex arithmetic are likely to be much faster than any implementation based on matrices, but the simplicity of the matrix exponential formulation discussed in this paper, and the fact that it can be computed using standard real or complex matrix arithmetic, without using a hypercomplex library, means that the matrix exponential formulation provides a very simple reference implementation which can be used for verification of the correctness of hypercomplex code. This is an important point, because verification of the correctness of hypercomplex FFT code is otherwise non-trivial. Verification of inversion is simple enough, but establishing that the spectral coefficients have the correct values is much less so. 


\section{A matlabß code}

We include here two short matLaBß functions for computing the forward transform given in (2), and (5), apart from the scale factors. The inverses can be computed simply by interchanging the input and output and negating the matrix roots of -1 . Neither function is coded for speed, on the contrary the coding is intended to be simple and easily verified against the equations.

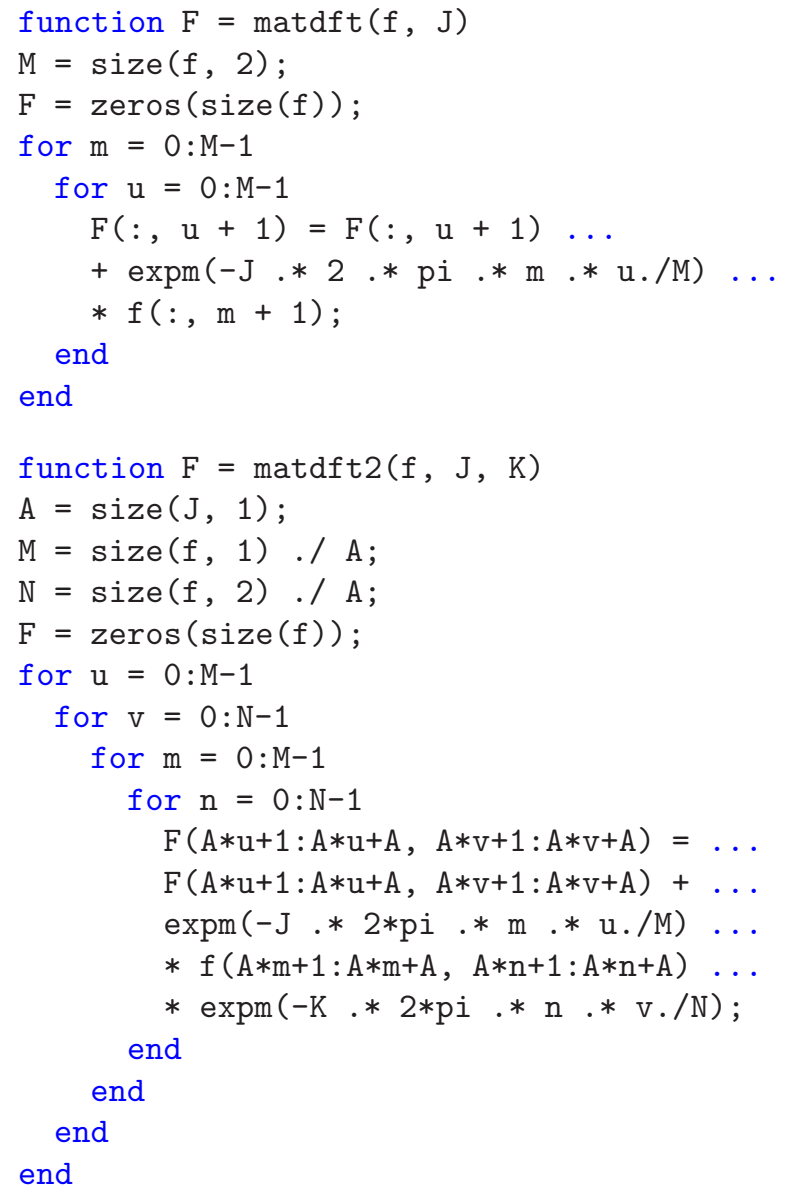

\section{References}

[1] R. N. Bracewell. The Fourier Transform and its Applications. McGraw-Hill, Boston, $3^{\text {rd }}$ edition, 2000.

[2] J. P. Ward. Quaternions and Cayley Numbers: Algebra and Applications, volume 403 of Mathematics and Its Applications. Kluwer, Dordrecht, 1997.

[3] B. P. Ickes. A new method for performing digital control system attitude computations using quaternions. AIAA Journal, 8(1):13-17, January 1970.

[4] R. R. Ernst, G. Bodenhausen, and A. Wokaun. Principles of Nuclear Magnetic Resonance in One and Two Dimensions. Oxford University Press, Oxford, 1987.

[5] M. A. Delsuc. Spectral representation of 2D NMR spectra by hypercomplex numbers. Journal of magnetic resonance, 77(1):119-124, March 1988.

[6] T. A. Ell. Hypercomplex Spectral Transformations. PhD thesis, University of Minnesota, 1992. 
[7] T. A. Ell. Quaternion-Fourier transforms for analysis of 2-dimensional linear time-invariant partialdifferential systems. In Proceedings of the 32nd IEEE Conference on Decision and Control, San Antonio, Texas, USA, 15 - 17 December 1993, volume 1-4, pages 1830-1841. IEEE, Control Systems Society, 1993.

[8] V. M. Chernov. Discrete orthogonal transforms with data representation in composition algebras. In Proceedings Scandinavian Conference on Image Analysis, pages 357-364, Uppsala, Sweden, 1995.

[9] T. Bülow. Hypercomplex Spectral Signal Representations for the Processing and Analysis of Images. PhD thesis, University of Kiel, Germany, 1999.

[10] T. Bülow and G. Sommer. Hypercomplex signals - a novel extension of the analytic signal to the multidimensional case. IEEE Trans. Signal Process., 49(11):2844-2852, November 2001.

[11] S. J. Sangwine and T. A. Ell. The discrete Fourier transform of a colour image. In J. M. Blackledge and M. J. Turner, editors, Image Processing II Mathematical Methods, Algorithms and Applications, pages 430-441, Chichester, 2000. Horwood Publishing for Institute of Mathematics and its Applications. Proceedings Second IMA Conference on Image Processing, De Montfort University, Leicester, UK, September 1998.

[12] Soo-Chang Pei, Jian-Jiun Ding, and Ja-Han Chang. Efficient implementation of quaternion Fourier transform, convolution, and correlation by 2-D complex FFT. IEEE Trans. Signal Process., 49(11):27832797, November 2001.

[13] Soo-Chang Pei, Ja-Han Chang, and Jian-Jiun Ding. Commutative reduced biquaternions and their Fourier transform for signal and image processing applications. IEEE Trans. Signal Process., 52(7):20122031, 2004.

[14] Julia Ebling and Gerik Scheuermann. Clifford Fourier transform on vector fields. IEEE Transactions on Visualization and Computer Graphics, 11(4):469-479, July/August 2005.

[15] T. A. Ell and S. J. Sangwine. Decomposition of 2D hypercomplex Fourier transforms into pairs of complex Fourier transforms. In Moncef Gabbouj and Pauli Kuosmanen, editors, Proceedings of EUSIPCO 2000, Tenth European Signal Processing Conference, volume II, pages 1061-1064, Tampere, Finland, 5-8 September 2000. European Association for Signal Processing.

[16] T. A. Ell and S. J. Sangwine. Hypercomplex Fourier transforms of color images. IEEE Trans. Image Process., 16(1):22-35, January 2007.

[17] S. J. Sangwine. Biquaternion (complexified quaternion) roots of -1. Advances in Applied Clifford Algebras, 16(1):63-68, June 2006.

[18] Salem Said, N. Le Bihan, and S. J. Sangwine. Fast complexified quaternion Fourier transform. IEEE Trans. Signal Process., 56(4):1522-1531, April 2008.

[19] Eckhard Hitzer and Rafał Abłamowicz. Geometric roots of -1 in Clifford algebras $C \ell_{p, q}$ with $p+q \leq 4$. Advances in Applied Clifford Algebras, 21(1):121-144, 2010. Published online 13 July 2010.

[20] Daniel Alfsmann, Heinz Göckler, S. J. Sangwine, and T. A. Ell. Hypercomplex algebras in digital signal processing: Benefits and drawbacks. In Proceedings of EUSIPCO 2007, 15th European Signal Processing Conference, pages 1322-6, Poznan, Poland, 3-7 September 2007. European Association for Signal Processing.

[21] Paul J. Nahin. Dr Euler's Fabulous Formula: Cures Many Mathematical Ills. Princeton University Press, 2006.

[22] Gene H. Golub and Charles F. van Loan. Matrix Computations. Johns Hopkins studies in the Mathematical Sciences. The Johns Hopkins University Press, Baltimore and London, third edition, 1996. 
[23] E. J. Borowski and J. M. Borwein, editors. Collins Dictionary of Mathematics. HarperCollins, Glasgow, 2nd edition, 2002.

[24] Stephen J. Sangwine and Nicolas Le Bihan. Quaternion Toolbox for Matlabß. http://qtfm.sourceforge.net/, 2005. Software library, licensed under the GNU General Public License.

[25] W. R. Hamilton. Researches respecting quaternions. First series (1843). In H. Halberstam and R. E. Ingram, editors, The Mathematical Papers of Sir William Rowan Hamilton, volume III Algebra, chapter 7, pages 159-226. Cambridge University Press, Cambridge, 1967. First published as [28].

[26] Pertti Lounesto, Risto Mikkola, and Vesa Vierros. CLICAL user manual. Research Report A248, Helsinki University of Technology, Institute of Mathematics, Espoo, Finland, August 1987.

[27] S. J. Sangwine. Fourier transforms of colour images using quaternion, or hypercomplex, numbers. Electronics Letters, 32(21):1979-1980, 10 October 1996.

[28] W. R. Hamilton. Researches respecting quaternions. Transactions of the Royal Irish Academy, 21:199296, 1848. 